\title{
I-PD Controller Tuning for Unstable System Using Bacterial Foraging Algorithm: A Study Based on Various Error Criterion
}

\author{
V. Rajinikanth ${ }^{1}$ and K. Latha ${ }^{2}$ \\ ${ }^{1}$ Department of Electronics and Instrumentation Engineering, St Joseph's College of Engineering, Chennai 600 119, India \\ ${ }^{2}$ Division of Avionics, Department of Aerospace Engineering, MIT Campus, Anna University, Chennai 600 044, India
}

Correspondence should be addressed to V. Rajinikanth, rajinisjceeie@gmail.com

Received 31 May 2011; Revised 21 August 2011; Accepted 2 October 2011

Academic Editor: Sebastian Ventura

Copyright (C) 2012 V. Rajinikanth and K. Latha. This is an open access article distributed under the Creative Commons Attribution License, which permits unrestricted use, distribution, and reproduction in any medium, provided the original work is properly cited.

\begin{abstract}
This paper proposes a novel method to tune the I-PD controller structure for the time-delayed unstable process (TDUP) using Bacterial Foraging Optimization (BFO) algorithm. The tuning process is focussed to search the optimal controller parameters $\left(K_{p}\right.$, $K_{i}, K_{d}$ ) by minimising the multiple objective performance criterion. A comparative study on various cost functions like Integral of Squared Error (ISE), Integral of Absolute Error (IAE), Integral of Time-weighted Squared Error (ITSE), and Integral of Time weighted Absolute Error (ITAE) have been attempted for a class of TDUP. A simulation study for BFO-based I-PD tuning has been done to validate the performance of the proposed method. The results show that the tuning approach is a model independent approach and provides enhanced performance for the setpoint tracking with improved time domain specifications.
\end{abstract}

\section{Introduction}

Proportional + Integral + Derivative (PID) controllers are widely used in various industrial applications in which setpoint tracking and disturbance rejection are necessary. This controller provides an optimal and robust performance for a wide range of operating conditions for stable, unstable and nonlinear processes. Based on the controller configuration (position of P, I, and D), the PID is classified as ideal PID, series PID, and parallel PID.

Since an ideal PID controller has practical difficulties due to its unrealizable nature, it is largely considered in academic studies. Parallel PID controllers are widely used in industries due to its easy accomplishment in analog or digital form. The major drawbacks of the basic parallel PID controllers are the effects of proportional and derivative kick. In order to minimize these effects, modified forms of parallel controller structures such as ID-P and I-PD are widely considered [1].

Time Delayed Unstable Processes (TDUP) considered in this work are widely observed in chemical process industries (exothermic stirred reactors with back mixing, pump with liquid storage tank, combined feed/effluent heat exchanger with adiabatic exothermic reaction, bioreactor, polymerization reactor, jacketed CSTR) [2]. Fine tuning of controller parameters for these systems is highly difficult than in open loop stable systems since (i) unstable processes are hard to stabilize due to unstable poles, (ii) the controller gains are limited by a minimum and maximum value based on the process time delay (ratio of process time delay to process time constant, that is, $d / \tau$ ratio). The increase in time delay " $d$ " in the process narrows down the limiting value and it restricts the performance of the closed loop system under control, (iii) unusual overshoot and/or inverse response due to the presence of zeros in the process model.

In control literature, many efforts have been attempted to design optimal and robust controllers for TDUP. Panda has proposed a synthesis method to design an Internal Model Controller-based PID (IMC-PID) controller for a class of time-delayed unstable process [2]. Padhy and Majhi have proposed a PI-PD controller design for unstable systems based on the phase and gain margin criteria [3]. Marchetti et al. have considered a relay-based identification and PID 
controller tuning [4]. Liu et al. have developed an analytical two-degree freedom setpoint tracking control scheme [5]. Shamsuzzoha and Lee have proposed a control scheme for enhanced disturbance rejection [6]. Chen et al. have discussed a setpoint weighted-PID controller tuning for timedelayed unstable system. It has been reported that, based on the setpoint weighting parameter, a simple PID-PD controller can be used to achieve basic and modified PID structures [7]. Apart from the above methods, a review on the methods of controller tuning for a class of time-delayed unstable system could be found in the book by Padma Sree and Chidambaram [8]. Most of these approaches require an approximated first or second-order transfer-function model with a time delay. In real time, the approximated model parameter may be changing or subject to uncertainty. The model-based controller tuning also requires complex computations to identify the controller parameters. To overcome this, it is necessary to use soft computing-based model independent controller tuning methods.

In recent years, evolutionary approach-based controller autotuning methods has attracted the control engineers and the researchers due to it is nonmodel-based approach, simplicity, high computational efficiency, easy implementation, and stable convergence [9-13]. In this paper, the I-PD controller tuning is proposed for unstable system using the Bacteria Foraging Optimization (BFO) algorithm introduced by Passino [14]. It is a biologically inspired computation technique based on mimicking the foraging activities of Escherichia coli (E. coli) bacteria, and it is successfully used in various engineering applications. The literature gives the application details of BFO in PID controller tuning for a class of stable systems $[15,16]$. Hybridization-based optimization techniques, such as Genetic Algorithm (GA) [17, 18] and Particle Swarm Optimization (PSO) [19-21] have also been used in PID controller tuning. The above methods are proposed for stable systems only. For stable systems, the overshoot and the error value will be very small and it supports the PID controller tuning efficiently. For unstable systems, the controller parameter tuning seems to be difficult task and is limited due to " $d / \tau$ " ratio. Since the basic PID controller will not provide the optimised parameter and this may require a modified PID structure such as I-PD.

Recently, the author has attempted BFO-based PID and I-PD tuning for a class of TDUP [22]. In this work, ISE minimization (single-objective function) is highly prioritized as a performance measure and it monitors the BFO until the controller parameters converge to a minimized value. From the work it has demonstrated that a BFO-based PID controller tuning can be performed for the unstable system when the " $d / \tau$ " ratio is below 0.2 . PID-based tuning results large overshoot which tends to increase the ISE value, when the $d / \tau$ ratio is greater than 0.2 . This phenomenon disrupts the convergence of BFO algorithm. In order to overcome the problem, an I-PD structure is employed to obtained better results. They have also presented a comparative study with the Particle-Swarm-Optimization-(PSO-) based controller tuning and classical controller tuning methods with a simulation study. The BFO-based controller tuning approach shows improved performance of the process in terms of time domain specification, error minimization, disturbance rejection, setpoint, and multiple setpoint tracking than the PSO and classical tuning methods.

In this work, a multiple-objective function-based $\mathrm{BFO}$ algorithm has been proposed for the controller parameter tuning for TDUP. Further, an attempt has been made by considering a TDUP with a zero. A comparative study on various cost functions such as ISE, IAE, ITSE, and ITAE, has been attempted. To evaluate the performance of the proposed method, a simulation study is carried out using a class of unstable system models.

The remaining part of the paper is organized as follows: an overview of bacterial foraging optimization algorithm is provided in Section 2, Section 3 presents the problem formulation and the cost function-based design of I-PD controller. Section 4 discusses the simulated results on different process models followed by the conclusion of the present research work in Section 5.

\section{Bacteria Foraging Optimization Algorithm}

Bacteria Foraging Optimization (BFO) algorithm is a new class of biologically inspired stochastic global search technique based on mimicking the foraging (methods for locating, handling, and ingesting food) behavior of E. coli bacteria. During foraging, a bacterium can exhibit two different actions: tumbling or swimming. The tumble action modifies the orientation of the bacterium. During swimming (chemotactic step), the bacterium will move in its current direction. Chemotactic movement is continued until a bacterium goes in the direction of positive-nutrient gradient. After a certain number of complete swims, the best half of the population undergoes reproduction, eliminating the rest of the population. In order to escape local optima, an elimination-dispersion event is carried out where some bacteria are liquidated at random with a very small probability and the new replacements are initialized at random locations of the search space $[14,16,19]$. Figure 1 shows the flow chart of the BFO algorithm.

The working principle for the bacterial foraging optimization algorithm can be defined as shown in Figure 1.

\section{I-PD Controller Tuning Procedure}

3.1. Problem Formulation. In process industries, PID controller is used to improve both the steady state as well as the transient response of the plant. Consider the closed loop control system as shown in Figure 2, where $G_{p}(s)$ is the process under control and $G_{c}(s)$ is the controller. The main objective of this system is to make $Y(s)=R(s)$. In this system, the controller continuously adjusts the value of " $U_{c}(s)$ " until the error " $E(s)$ " is zero irrespective of the disturbance signal " $D(s)$."

For practical applications, the term " $G_{c}(s)$ " can be replaced by a simple PID controller or a modified structure PID controller.

(i) Let, $G_{c}(s)$ has the noninteracting $\left(K_{p}, K_{i}, K_{d}\right.$ works independently on error signal) form of parallel PID structure as shown in Figure 3. 


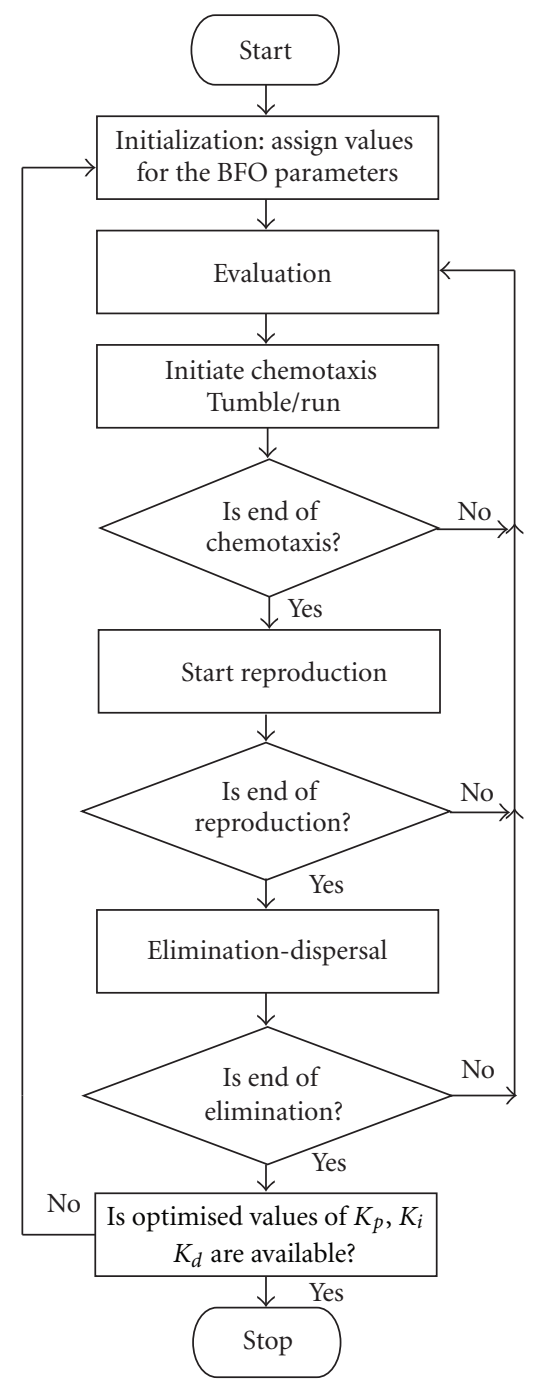

FIGURE 1: Flow chart for bacterial foraging algorithm.

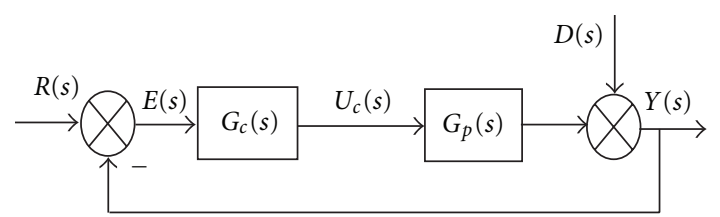

FIGURE 2: Block diagram of the closed loop control system.

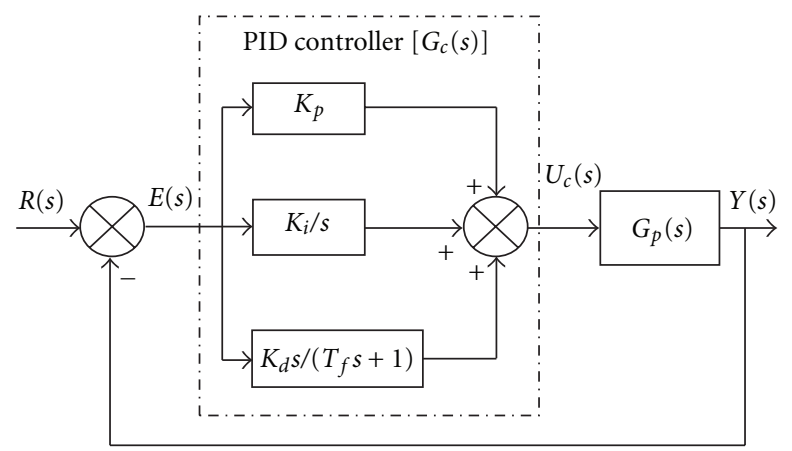

FIGURE 3: Structure of parallel PID control system.

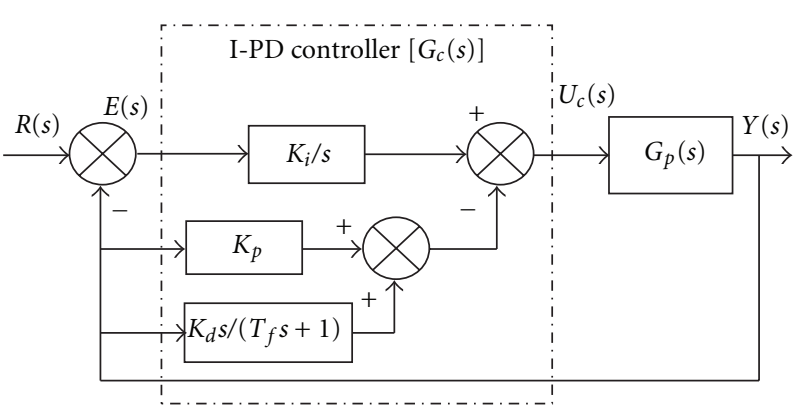

FigURE 4: Structure of parallel I-PD control system.

Mathematical representation of parallel form of PID controller is given in (1):

$$
G_{c}(s)=\left(K_{p}+\frac{K_{i}}{s}+\frac{K_{d} s}{T_{f} s+1}\right),
$$

where $T_{f}$ if the filter time constant. $T_{f}=T_{d} / N ; T_{d}=$ Derivative controller time constant $\left(K_{d} / K_{p}\right) ; N=$ derivative filter constant. For this study it is assigned as 10.

The output signal from the controller is

$$
U_{c}(s)=K_{p} e(t)+K_{i} \int_{0}^{T} e(t) d t+\left(\frac{K_{d}}{T_{f} s+1}\right) \frac{d e(t)}{d t} .
$$

In this structure, a step change in the reference input " $R(s)$ " will cause an immediate spiky change in the control signal " $U_{c}(s)$." This abrupt change in the controller output is represented as the proportional and/or derivative kick. These kick effects rapidly change the command signal to the actuator which controls the entire operation of the plant $\left(G_{p}(s)\right)[1]$. To overcome this drawback, a modified controller structure knows as I-PD is considered in this study.

(ii) The noninteracting form of I-PD controller structure is shown in Figure 4.

The output signal from the I-PD controller is

$$
U_{c}(s)=K_{i} \int_{0}^{T} e(t) d t-\left[K_{p} y(t)+K_{d} \frac{d y(t)}{d t}\right] .
$$

In this structure, the integral term $\left(K_{i}\right)$ responds on error signal " $e(t)$." An abrupt change in the reference input " $R(s)$ " will not affect the proportional $\left(K_{p}\right)$ and derivative $\left(K_{d}\right)$ terms, since these two terms works on the process output " $y(t)$." The I-PD is a modified form of a parallel PID structure and is always preferred in industries, where a smooth set point tracking is required.

3.2. Cost Function. A generalized closed loop response of a system is shown in Figure 5. For closed loop systems, the main objective of the controller is to make the peak overshoot $\left(M_{p}\right)$, settling time $\left(t_{s}\right)$ and final steady state error $\left(E_{\mathrm{ss}}\right)$, as small as possible. During the optimization search, along with the cost function it is necessary to assign the essential values for $M_{p}, t_{s}$ and $E_{\mathrm{ss}}$.

(i) " $M_{p}$ " is the difference between the reference input " $R(s)$ " and the maximum process output " $Y(s)$." 


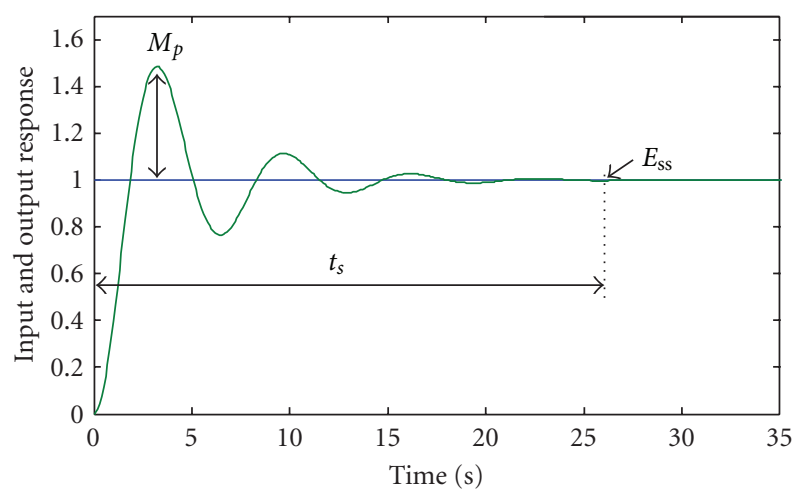

- Reference input " $R(s)$ "

— Process output " $Y(s)$ "

FIGURE 5: Closed loop response of the system.

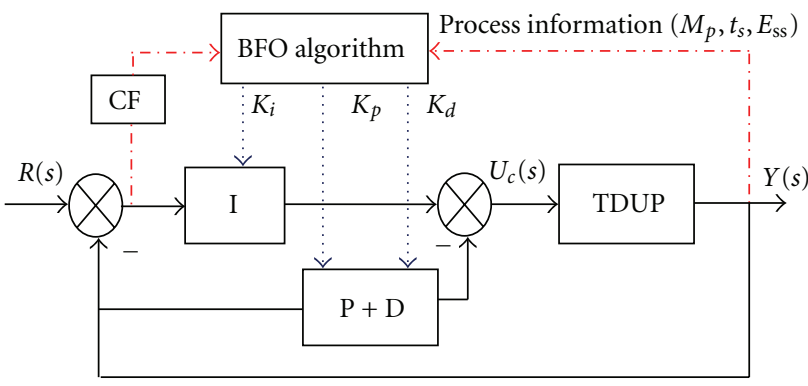

Figure 6: BFO-based I-PD controller tuning.

(ii) " $t_{s}$ " is the time required for the process output to reach a final steady state value.

(iii) " $E_{\mathrm{ss}}$ " is the value of error " $e(t)$ " when time " $t$ " tends to infinity.

The Cost Function (CF) guides the algorithm to search the optimised controller parameters. Equation (4) shows the normally considered CF to evaluate the performance of the closed loop system:

$$
\begin{aligned}
\text { IAE } & =\int_{0}^{T}|e(t)| d t=\int_{0}^{T}|r(t)-y(t)| d t, \\
\text { ISE } & =\int_{0}^{T} e^{2}(t) d t=\int_{0}^{T}[r(t)-y(t)]^{2} d t, \\
\text { ITAE } & =\int_{0}^{T} t|e(t)| d t=\int_{0}^{T} t|r(t)-y(t)| d t, \\
\text { ITSE } & =\int_{0}^{T} t e^{2}(t) d t=\int_{0}^{T} t[r(t)-y(t)]^{2} d t,
\end{aligned}
$$

where $e(t)=$ error, $r(t)=$ reference input (set point), and $y(t)=$ process output.

The performance criterion in the proposed method is expressed as,

$$
\begin{aligned}
J_{1}\left(K_{p}, K_{i}, K_{d}\right)= & \left(w_{1} \cdot \mathrm{CF}\right)+\left(w_{2} \cdot M_{p}\right) \\
& +\left(w_{3} \cdot t_{s}\right)+\left(w_{4} \cdot E_{\mathrm{ss}}\right),
\end{aligned}
$$

where $w_{1} \cdots w_{4}$ are weighting functions for the performance index (range is from $0-1$ ), CF is the error criterion from the error detector, and $M_{p}, t_{s}$, and $E_{s s}$ are additional performance index obtained from the process output.

The performance criterion presented in (5) is a multiobjective criterion and has four terms accompanied by a weighting factor " $w$," and it should be set based on the priority level. If the parameter " $J_{1}\left(K_{p}, K_{i}, K_{d}\right)$ " does not converge with an optimal value during the entire search, then the exploration is reinitialized with " $w_{1}$ " alone by making other weights as zero.

3.3. Design of I-PD Controller Using BFO. The controller tuning process is to find the optimal values for $K_{p}, K_{i}$, and $K_{d}$ from the search space that minimizes the objective function (5). Figure 6 illustrates the basic block diagram for I-PD controller tuning using $\mathrm{BFO}$ algorithm. In this, the performance criterion " $J_{1}\left(K_{p}, K_{i}, K_{d}\right)$ " guides the optimization algorithm to get appropriate value for the controller parameters.

Prior to the optimization search, it is necessary to assign the following algorithm parameters.

Dimension of search space is three; number of bacteria is chosen as ten; number of chemotaxis step is set to five; number of reproduction steps and length of a swim is considered as four; number of elimination-dispersal events is two; number of bacteria reproduction is assigned as five; probability for elimination dispersal has a value of 0.2 .

In the literature, there is no guide line to allot the tuning parameters for the $\mathrm{BFO}$ algorithm.

In this study, before proceeding with the BFO-based I-PD controller tuning, the following values are assigned.

(i) The three dimensional search space is defined as:

$$
\begin{aligned}
& K_{p}: \min -60 \% \text { to } \max +60 \% \\
& K_{i}: \min -30 \% \text { to } \max +30 \% ; \\
& K_{d}: \min -30 \% \text { to } \max +30 \% .
\end{aligned}
$$

If the search does not converge with an optimal $K_{p}, K_{i}, K_{d}$ values, increase the search range by $10 \%$ and begin a new search.

(ii) The maximum overshoot $\left(M_{p}\right)$ range is selected as $50 \%$ of the reference signal. The overshoot in the process output is limited by inserting a rate and limiter block between the controller and the TDUP.

(iii) The steady state error $\left(E_{\mathrm{ss}}\right)$ in the process output is assigned as zero.

(iv) There is no guideline to specify the values for CF and settling time $\left(t_{s}\right)$. The " $t_{s}$ " is preferred as $25 \%$ of the maximum simulation time. The simulation time should be selected based on the process time delay (d).

(v) For each process example, five trials with a particular $\mathrm{CF}$ are carried out and the finest set of values among the trials are selected as the optimized controller parameter set. 


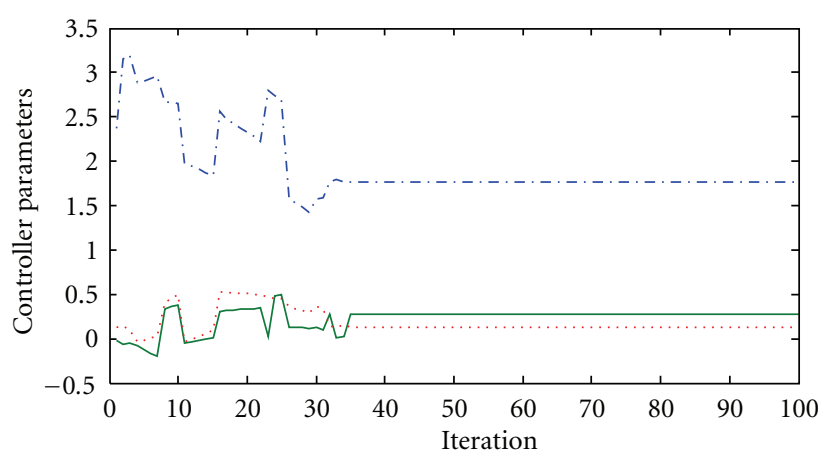

(a)

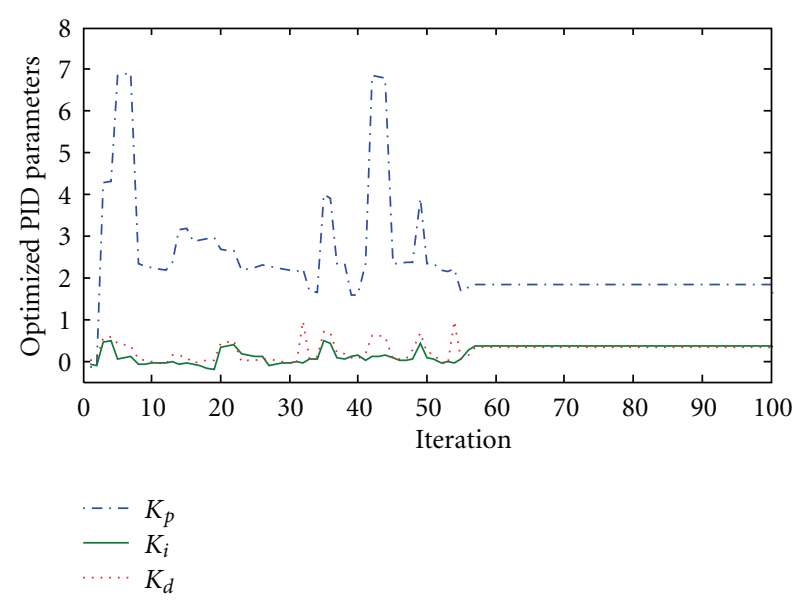

(c)

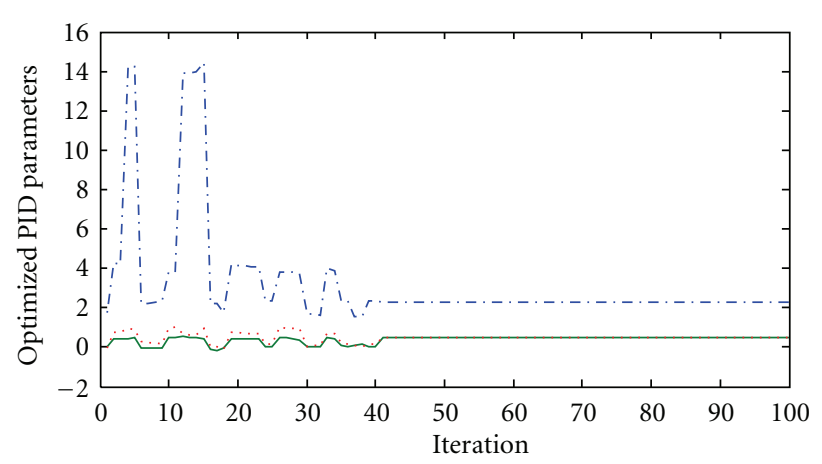

(b)

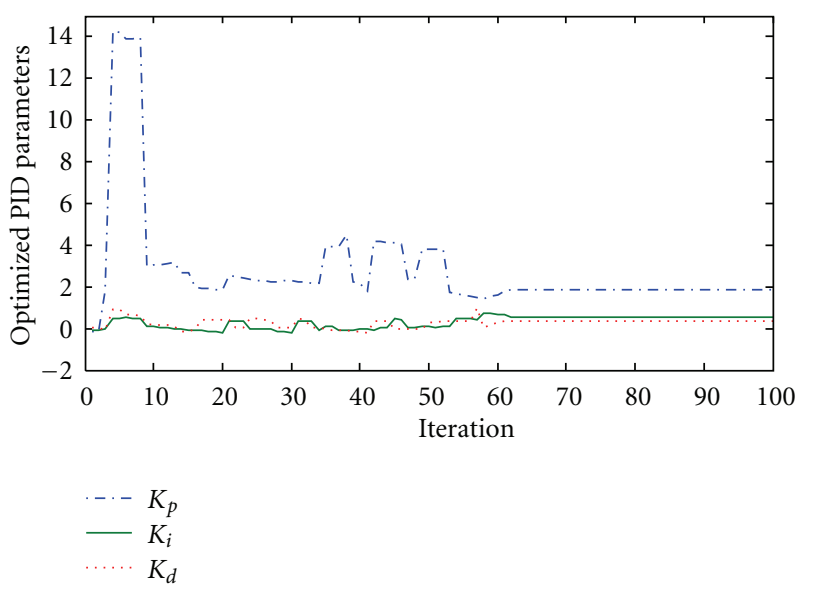

(d)

FIGURE 7: Final convergence of controller parameters with (a) ISE-, (b) IAE-, (c) ITAE-, and (d) ITSE-guided BFO algorithm.

TABLE 1: Optimised controller values.

\begin{tabular}{lcccc}
\hline Method & Iteration number & $K_{p}$ & $K_{i}$ & $K_{d}$ \\
\hline ISE I-PD & 35 & 1.7726 & 0.2809 & 0.1339 \\
IAE I-PD & 41 & 2.2530 & 0.4752 & 0.4628 \\
ITAE I-PD & 57 & 1.8399 & 0.3722 & 0.3194 \\
ITSE I-PD & 62 & 1.8874 & 0.5199 & 0.3836 \\
\hline
\end{tabular}

(vi) A unity reference signal is considered for all the process models, (that is, $R(s)=1$ ).

\section{Results and Discussions}

To study the closed loop performance of the TDUP with BFO tuned I-PD controller, mathematical model of the processes from literature are considered.

Example 1. The first order system with the following transfer function model is considered:

$$
G_{p}(s)=\frac{e^{-d s}}{\tau s-1}=\frac{e^{-0.4 s}}{s-1} .
$$

This process has an unstable pole with process time constant $(\tau)=1$ and time delay $(d)=0.4$. For the above process " $d / \tau$ " is 0.4 .
The BFO-based I-PD controller tuning is proposed for the system as in Figure 6. The final convergence of the controller parameters are shown in Figure 7 and the optimised $K_{p}, K_{i}, K_{d}$ values are tabulated in Table 1 . The result shows that the ISE-based tuning has less number of iteration compared to other methods.

The process model (6) is initially tested with the parallel PID controller (Figure 3 ) with the optimal values given in Table 1. From Figure 8(a), it is noted that the overshoot observed in the process output is very large and the controller output illustrates a large spike as in Figure 8(b).

An enlarged view of the controller output " $U_{c}(s)$ " is presented in Figure 9. When the process is excited with a reference signal, the controller will produce proportional and derivative kick initially. When the output reaches the final steady state value, the abrupt change in the controller signal vanishes slowly, and finally it reaches a smooth stable value. The initial part of the controller output is the cause for the process overshoot " $M_{p}$."

The given process model is then proceeded with an I-PD controller (Figure 4) with Table 1 parameters. Figures 10(a) and 10 (b) show the process and controller outputs respectively. The present method provides enhanced performance compared to the basic PID controller.

The regulatory response is then studied with a load disturbance of 0.1 (10\% of setpoint) introduced at $30 \mathrm{sec}$. 
TABLE 2: Performance comparisons for Example 1 with I-PD controller.

\begin{tabular}{lcccccccccccc}
\hline Method & \multicolumn{1}{c}{ Reference tracking } & \multicolumn{4}{c}{ Disturbance rejection } \\
\hline & ISE & IAE & ITAE & ITSE & $t_{r}(s)$ & $M_{p}$ & $t_{s}(s)$ & $E_{\mathrm{ss}}$ & ISE & IAE & ITAE & ITSE \\
\hline ISE I-PD & 1.959 & 2.750 & 4.989 & 3.582 & 4.50 & 0 & 8.20 & 0 & 2.051 & 3.234 & 20.36 & 19.62 \\
IAE I-PD & 1.813 & 2.637 & 4.687 & 3.225 & 4.60 & 0 & 8.25 & 0 & 1.872 & 3.938 & 46.79 & 37.85 \\
ITAE I-PD & 1.793 & 2.441 & 3.889 & 2.923 & 3.65 & 0.0323 & 9.55 & 0 & 1.926 & 3.151 & 19.44 & 17.03 \\
ITSE I-PD & 1.565 & 2.230 & 3.675 & 2.681 & 2.85 & 0.1039 & 8.25 & 0 & 1.700 & 2.935 & 17.92 & 15.92 \\
\hline
\end{tabular}

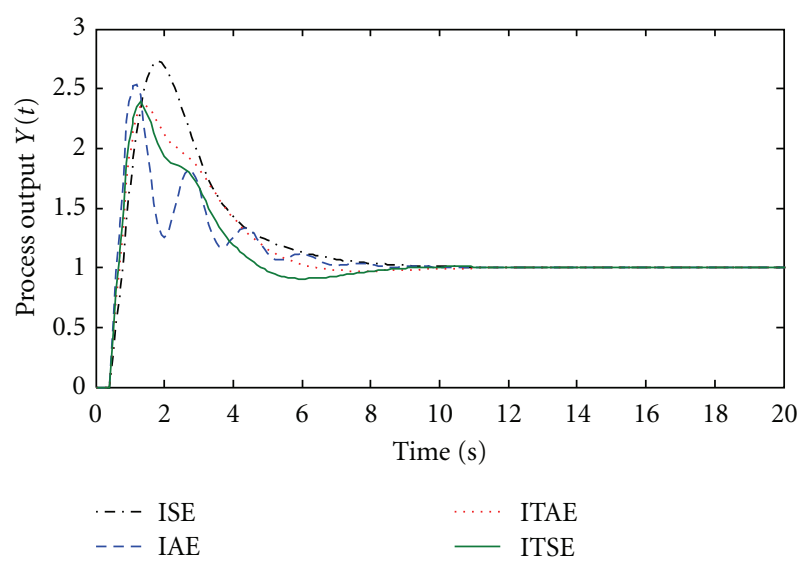

(a)

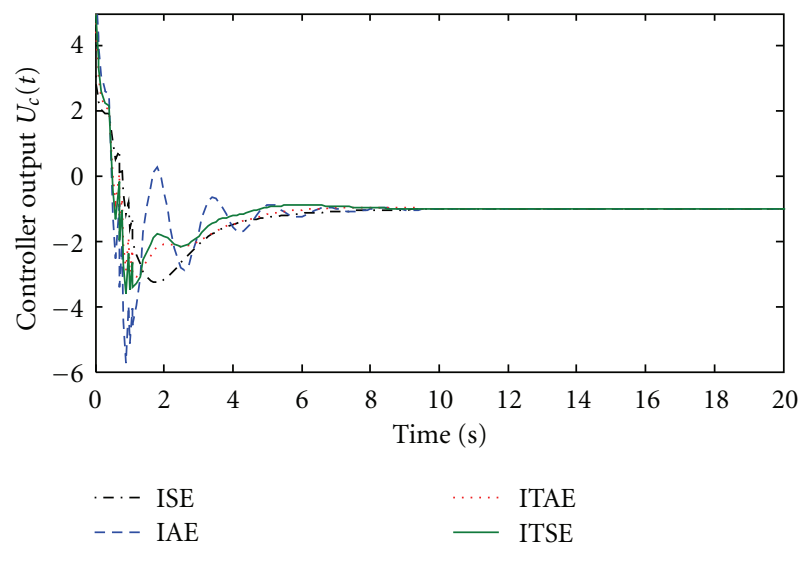

(b)

FIGURE 8: PID controller performance for Example 1, (a) process output, (b) controller output.

TABle 3: Optimised $K_{p}, K_{i}$, and $K_{d}$ values for Example 2.

\begin{tabular}{lcccc}
\hline Method & Iteration number & $K_{p}$ & $K_{i}$ & $K_{d}$ \\
\hline ISE I-PD & 174 & 1.3394 & 0.0479 & 0.6195 \\
IAE I-PD & 189 & 1.9037 & 0.1173 & 0.9032 \\
ITAE I-PD & 238 & 1.5518 & 0.0968 & 0.8471 \\
ITSE I-PD & 251 & 1.5892 & 0.1204 & 0.8949 \\
\hline
\end{tabular}

From Figure 11, it is observed that the I-PD controller tuned with ISE, ITAE, and ITSE offers a stable response. The IAE tuned controller provides an oscillatory response for load disturbance rejection.

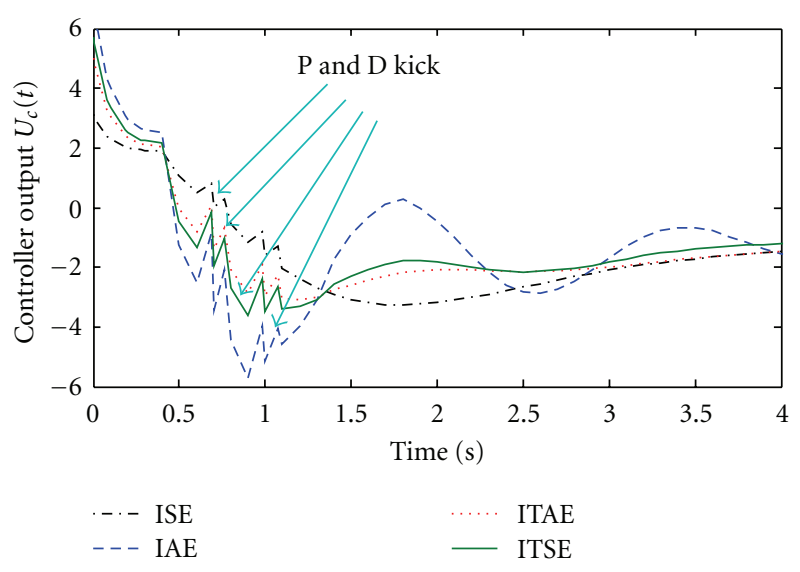

FIGURE 9: The effect of proportional and derivative kick in $U_{c}(t)$.

Table 2 presents the performance of the I-PD controller for setpoint tracking and load disturbance rejection. The overshoot produced by the ITSE tuned controller (ITSE IPD) has a large overshoot compared to other methods. But the final error by this method is modest compared with the ISE, IAE, and ITSE.

Example 2. The second order TDUP with the following transfer function is considered. It has one unstable pole and a stable pole:

$$
G_{p}(s)=\frac{\exp ^{-s}}{(2 s-1)(0.5 s+1)}
$$

For this process delay time $(d)$ is unity. The PID-tuning scheme previously proposed for the above model is presented in the literature [7].

For this model, the BFO-based I-PD is proposed with a bacteria size of 18 and the other values as given in Section 3.3. The time delay $(d)$ for the system is 1 , hence the simulation time is fixed as $200 \mathrm{sec}$. The trials are executed with each CF and the optimal values are tabulated in Table 3. ISE-based method offers the optimal values with less convergence time than IAE, ITAE, and ITSE.

Figure 12 and Table 4 depict the reference tracking performance of the process model with I-PD structure. The overshoot produced by the ISE (ISE I-PD) is large. The response by the IAE is more oscillatory since the settling time of the system is large. The final steady state error by all the methods are zero. ITAE-based I-PD has a lesser overshoot. 


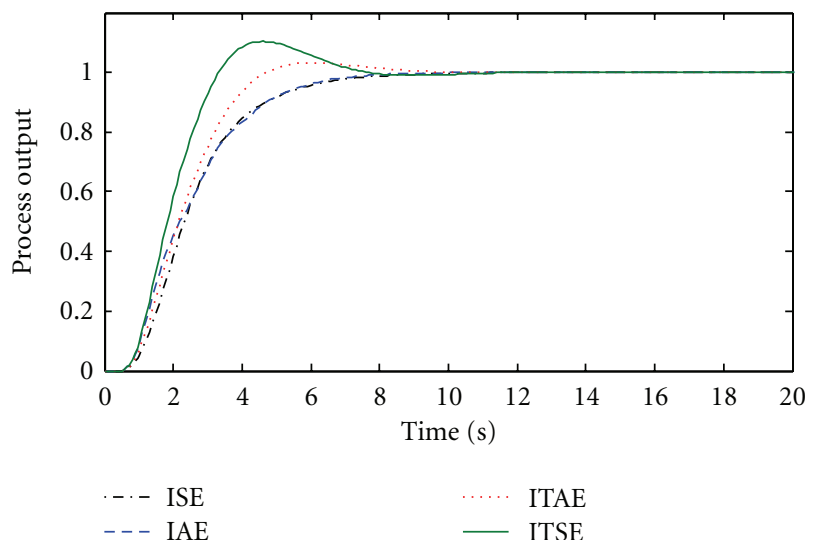

(a)

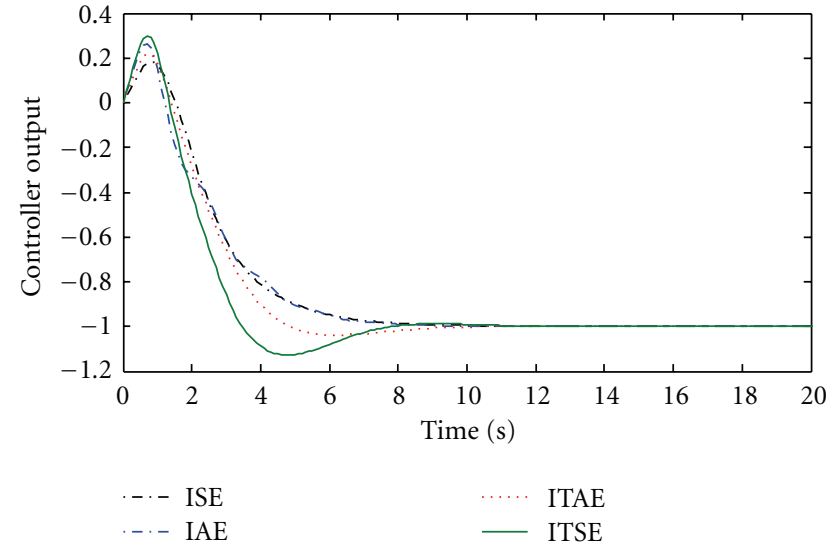

(b)

FIGURE 10: I-PD controller performance, (a) process output, (b) controller output.

TABle 4: Performance evaluation for Example 2.

\begin{tabular}{lcccccccc}
\hline \multirow{2}{*}{ Method } & \multicolumn{9}{c}{ Reference tracking } & \multicolumn{2}{c}{${ }^{2}$} & $t_{s}(s)$ & \\
& ISE & IAE & ITAE & ITSE & $t_{r}(s)$ & 11.1 & 0.0343 & 27.9 \\
\hline ISE I-PD & 5.775 & 7.485 & 33.73 & 24.71 & 13.7 & 0.0003 & 40.2 \\
IAE I-PD & 4.852 & 7.706 & 47.50 & 39.42 & 8.59 & 0.0002 & 19.1 & 0 \\
ITAE I-PD & 4.541 & 5.702 & 18.62 & 17.55 & 7.33 & 0.0009 & 18.5 \\
ITSE I-PD & 4.184 & 5.239 & 10.00 & 9.681 & & 0 \\
\hline
\end{tabular}

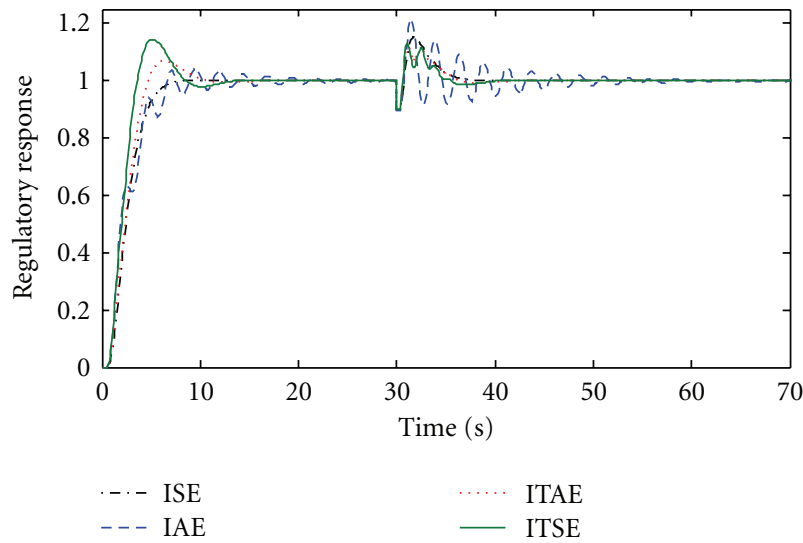

FIGURE 11: Load disturbance rejection for Example 1 with I-PD controller.

TABLE 5: Optimised controller parameters.

\begin{tabular}{lcccc}
\hline Method & Iteration number & $K_{p}$ & $K_{i}$ & $K_{d}$ \\
\hline ISE I-PD & 153 & 1.5309 & 0.0349 & 1.1747 \\
IAE I-PD & 169 & 1.7045 & 0.0596 & 2.1298 \\
ITAE I-PD & 378 & 1.8996 & 0.0807 & 1.5813 \\
ITSE I-PD & 391 & 2.0109 & 0.1052 & 1.8934 \\
\hline
\end{tabular}

The overall response of ITAE-based tuning outperforms the remaining methods considered in this study.

The robustness of the I-PD controller is analysed by changing the delay time of the process model. The controller

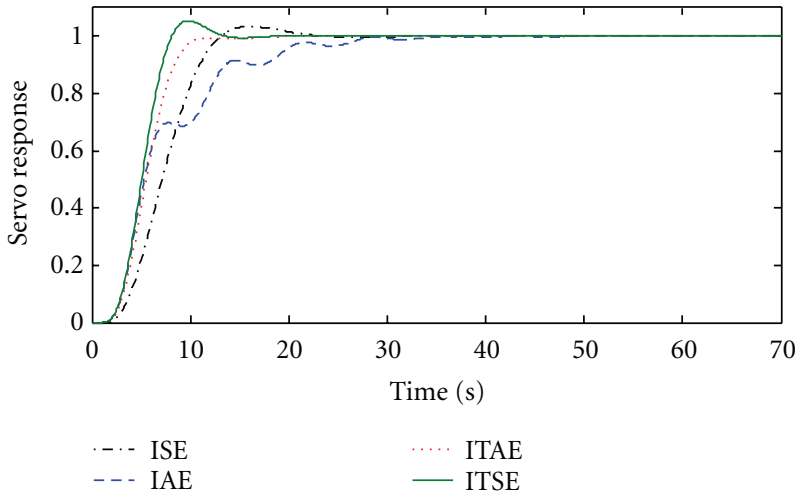

FIGURE 12: Servo response for Example 2 with I-PD controller.

values by ISE are employed to test the controller performance.

(i) $-50 \%$ change is applied in the delay $(d=0.5 \mathrm{sec})$. The process model is as in (8):

$$
G_{p}(s)=\frac{\exp ^{-0.5 s}}{(2 s-1)(0.5 s+1)} .
$$

The above model has been studied by the researchers and classically tuned controller settings are existing in the literature $[2,8]$.

(ii) $+25 \%$ change in the delay $(d=1.25)$ is tested with the I-PD and PID structures and the results are presented in Figures 13 and 14, respectively. 
TABLE 6: Reference tracking performance.

\begin{tabular}{lcccccccc}
\hline \multirow{2}{*}{ Method } & \multicolumn{9}{c}{ Reference tracking } & \multicolumn{2}{c}{$M_{p}$} & $t_{s}(s)$ & $E_{\text {ss }}$ \\
& ISE & IAE & ITAE & ITSE & $13)$ & 23.2 & 0.0134 & 67.8 \\
ISE I-PD & 11.70 & 15.42 & 147.9 & 162.8 & 19.0 & 0.0189 & 62.5 \\
IAE I-PD & 9.899 & 13.48 & 118.1 & 137.5 & 15.9 & 0.0121 & 68.8 \\
ITAE I-PD & 8.591 & 11.58 & 95.06 & 124.9 & 14.0 & 0.0184 & 75.5 \\
ITSE I-PD & 7.841 & 10.85 & 87.12 & 115.2 & 0 & 0 \\
\hline
\end{tabular}

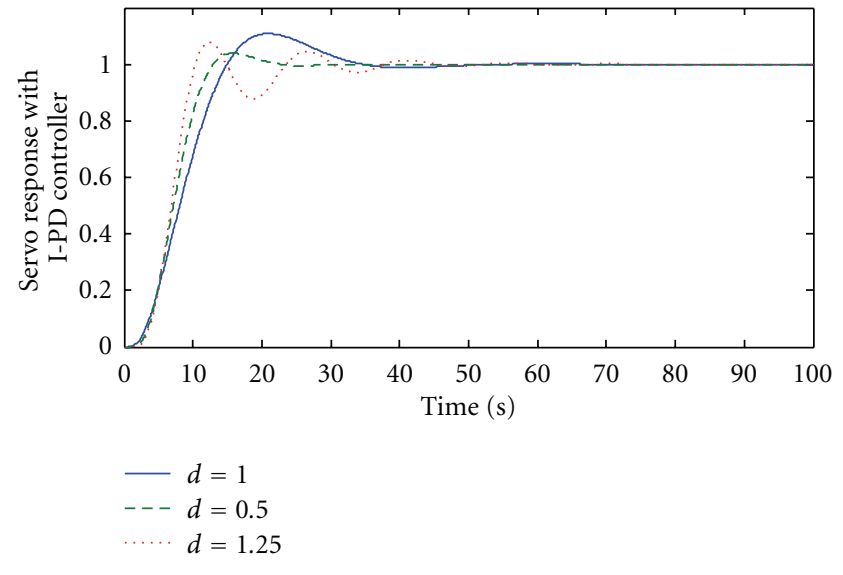

FIGURE 13: BFO-based I-PD controller tuning.

TABLE 7: Optimised parameters for CSTR model.

\begin{tabular}{lcccc}
\hline Method & Iteration number & $K_{p}$ & $K_{i}$ & $K_{d}$ \\
\hline ISE I-PD & 439 & 1.913 & 0.0412 & 0.1094 \\
IAE I-PD & 493 & 2.4571 & 0.0509 & 0.2109 \\
ITAE I-PD & 526 & 1.8662 & 0.0258 & 0.2168 \\
ITSE I-PD & 558 & 1.6984 & 0.0195 & 0.1107 \\
\hline
\end{tabular}

From Figures 13 and 14, the increase in the time delay may increase the overshoot of the system. When the time delay is large, the system condition will move from stable state to unstable state. From the result, it is noted that the proposed BFO-based tuning provides the optimal controller parameters and it works well for the system with a time delay uncertainty.

Example 3. A third order unstable process with delay studied by Chen et al. [7] is considered. The model has one unstable pole and two stable poles:

$$
G_{p}(s)=\frac{\exp ^{-0.5 s}}{(5 s-1)(0.5 s+1)(2 s+1)} .
$$

The BFO-tuned controller gains and the final iteration numbers are provided in Table 5. The ISE-based controller parameter search value is converging at 153rd iteration.

Figure 15 and Table 6 show the servo response of the process for unity step input $(R(s)=1)$. The overshoots produced by these methods are negligible. The BFO-tuned IPD controller provides an enhanced reference tracking with improved time domain specifications.

Example 4. Continuous Stirred Tank Reactor (CSTR) with nonideal mixing considered by Liou and Yu-Shu [23] has the transfer function model:

$$
\begin{gathered}
\frac{d C}{d t}=\frac{n Q}{m V}\left(C_{f}-C\right)-\left[\frac{K_{1} C}{\left(K_{2} C+1\right)^{2}}\right], \\
n C+(1-n) C_{f}=C_{e}, \\
\text { at } t=0, \quad C=C_{0} .
\end{gathered}
$$

In this process the nonideal mixing is described by Cholette's model. The values of the operating conditions are given by flow rate $(Q)=0.03333 \mathrm{lit} / \mathrm{sec}$; volume $(V)=1 \mathrm{lit}, K_{1}=$ $10 \mathrm{lit} / \mathrm{s} ; K_{2}=10 \mathrm{lit} / \mathrm{mol}, n=m=0.75$; concentration $\left(C_{f}\right)=3.288 \mathrm{~mol} / \mathrm{lit} ; C_{e}=1.8 \mathrm{~mol} / \mathrm{lit}$. Measurement delay is $20 \mathrm{sec}$. Linearization of the model equation around the operating condition " $C=1.304 \mathrm{~mol} /$ lit" gives the following unstable transfer function model:

$$
G_{p}(s)=\frac{\Delta C_{e}(s)}{\Delta C_{f}(s)}=\frac{2.21(1+11.133 s) \exp ^{-20 s}}{(98.3 s-1)} .
$$

The process model has one unstable pole and a stable zero (numerator "s" value). The unstable system with a zero may produce a large overshoot or inverse response. The time delay " $d$ " for the system is $20 \mathrm{sec}$.

The optimization search is initiated with the following algorithm parameters.

Number of bacteria is chosen as 25; number of chemotactic steps is set to ten; number of reproduction steps and length of a swim is considered as ten; number of eliminationdispersal events is five; number of bacteria reproduction is assigned as ten; probability for elimination dispersal has a value of 0.3 .

In the performance criterion (5), the overshoot " $M_{p}$ " is removed by assigning the weighting function value with a zero $\left(w_{2}=0\right)$. During the simulation study, the overshoot in the process output is controlled with a rate and the limiter unit. For this process, the simulation time is chosen as $500 \mathrm{~s}$.

The BFO I-PD parameters converge at 439th iteration for ISE and 558th iteration for ITSE. The controller gains and the performance measure are presented in Tables 7 and 8 , respectively. The servo response of the process model with unit step input is illustrated in Figure 16. The ITSE IPD controller provides a smooth performance for reference 
TABLE 8: Performance index for the CSTR model.

\begin{tabular}{|c|c|c|c|c|c|c|c|c|}
\hline \multirow{2}{*}{ Method } & \multicolumn{8}{|c|}{ Reference tracking } \\
\hline & ISE & IAE & ITAE & ITSE & $t_{r}(s)$ & $M_{p}$ & $t_{s}(s)$ & $E_{\mathrm{ss}}$ \\
\hline ISE I-PD & 137.8 & 279.3 & 82112.9 & 67391.4 & 59.4 & 0.1409 & 287 & 0 \\
\hline IAE I-PD & 135.3 & 275.2 & 81416.4 & 66942.7 & 53.7 & 0.0681 & 396 & 0 \\
\hline ITAE I-PD & 113.3 & 253.0 & 80461.3 & 59448.0 & 94.7 & 0.0431 & 380 & 0 \\
\hline ITSE I-PD & 106.9 & 244.2 & 79848.5 & 57489.3 & 111.3 & 0.0407 & 320 & 0 \\
\hline
\end{tabular}

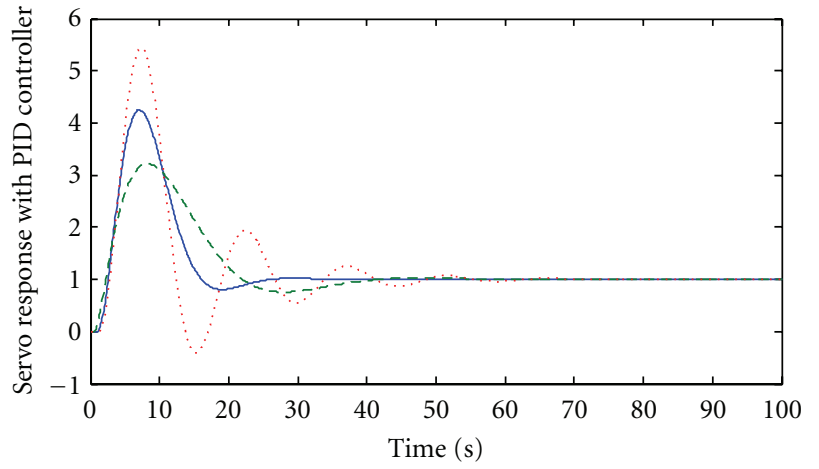

$\begin{aligned}-d & =1 \\ ---d= & 0.5 \\ \cdots-d & =1.25\end{aligned}$

FIGURE 14: BFO-based PID controller tuning.

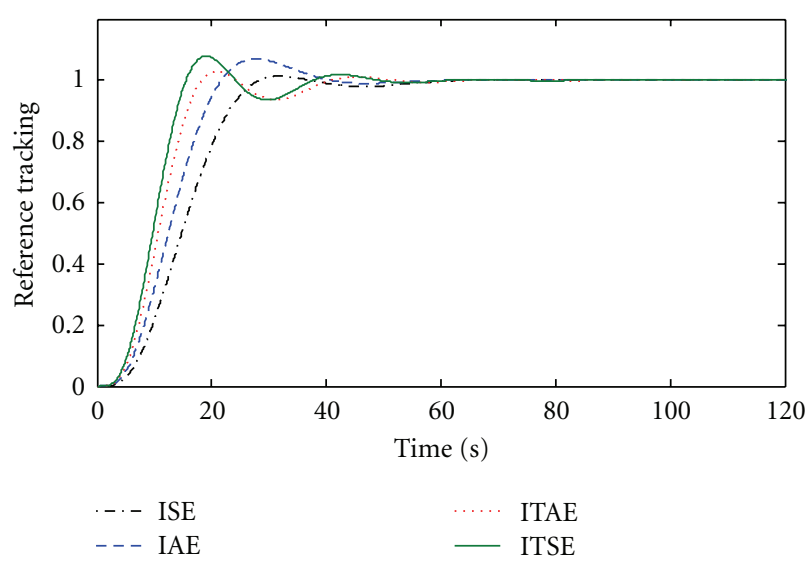

FIGURE 15: Servo response for Example 3 with BFO-based I-PD controller.

tracking and it outperforms the performance by other methods analysed in this study.

\section{Conclusion}

In this work, an attempt has been made for tuning an I-PD controller structure for a class of unstable process models using Bacterial Foraging Optimization (BFO) algorithm with minimizing the multiple objective performance criterion. A comparative study with Integral of Squared Error (ISE), Integral of Absolute Error (IAE), Integral of

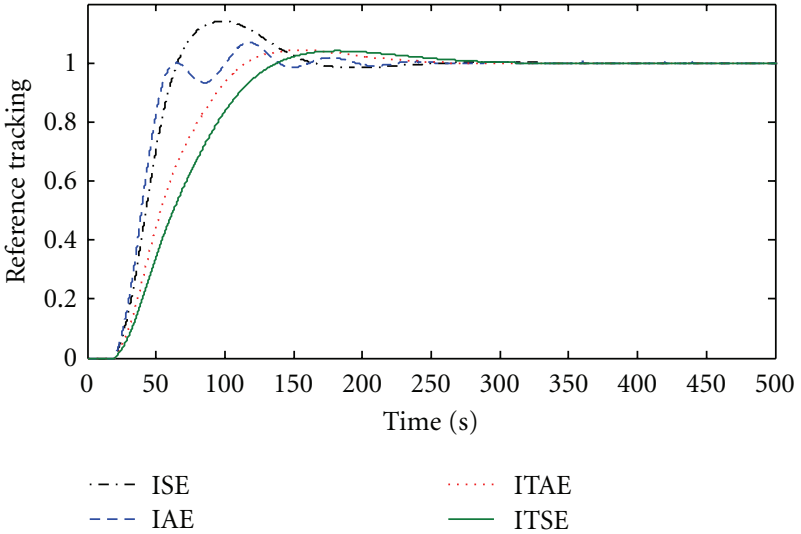

FIGURE 16: Reference tracking performance of CSTR model.

Time weighted Squared Error (ITSE), and Integral of Time weighted Absolute Error (ITAE) have been discussed. The ISE based method provides the optimized value with a small iteration time than the IAE, ITAE, and ITSE. Hence, ISEbased controller tuning can be employed for unstable systems to obtain optimal controller settings compared to other methods. The I-PD structure provides an enhanced setpoint tracking performance with minimal cost function. It also provides improved time domain specifications and robust performance for the system with time delay uncertainty.

\section{Nomenclature}

$R(s): \quad$ Reference/input signal for the closed loop system

$Y(s)$ : Process output

$U_{c}(s)$ : Controller output

$E(s): \quad$ Error signal $=R(s)-Y(s)$

$D(s)$ : External disturbance

$r(t): \quad$ Laplace inverse of $R(s)$ that is, $L^{-1}[R(s)]$

$y(t): \quad L^{-1}[Y(s)]$

$e(t): \quad L^{-1}[E(s)]=r(t)-y(t)$

$K_{p}: \quad$ Proportional gain

$K_{i}: \quad$ Integral gain

$K_{d}: \quad$ Derivative gain

$t_{r}: \quad$ Rise time

$t_{s}$ : $\quad$ Settling time

$M_{p}$ : Over shoot

$E_{\mathrm{ss}}: \quad$ Steady state error

$K_{1}, K_{2}$ : CSTR constants.

$n, m$ : Constants 


\section{Subscripts}

$d$ : Derivative

$i$ : Integral

$p$ : Proportional

$f:$ Feed.

\section{References}

[1] M. A. Johnson and M. H. Moradi, PID Control: New Identification and Design Methods, chapter 2, Springer, London, UK, 2005.

[2] R. C. Panda, "Synthesis of PID controller for unstable and integrating processes," Chemical Engineering Science, vol. 64, no. 12, pp. 2807-2816, 2009.

[3] P. K. Padhy and S. Majhi, "Relay based PI-PD design for stable and unstable FOPDT processes," Computers \& Chemical Engineering, vol. 30, no. 5, pp. 790-796, 2006.

[4] G. Marchetti, C. Scali, and D. R. Lewin, "Identification and control of open-loop unstable processes by relay methods," Automatica, vol. 37, no. 12, pp. 2049-2055, 2001.

[5] T. Liu, W. Zhang, and D. Gu, "Analytical design of two-degreeof-freedom control scheme for open-loop unstable processes with time delay," Journal of Process Control, vol. 15, no. 5, pp. 559-572, 2005.

[6] M. Shamsuzzoha and M. Lee, "Enhanced disturbance rejection for open-loop unstable process with time delay," ISA Transactions, vol. 48, no. 2, pp. 237-244, 2009.

[7] C. C. Chen, H. P. Huang, and H. J. Liaw, "Set-point weighted PID controller tuning for time-delayed unstable processes," Industrial and Engineering Chemistry Research, vol. 47, no. 18, pp. 6983-6990, 2008.

[8] R. Padma Sree and M. Chidambaram, Control of Unstable Systems, Narosa Publishing House, New Delhi, India, 2006.

[9] M. Zamani, N. Sadati, and M. K. Ghartemani, "Design of an $H \infty$, PID controller using particle swarm optimization," International Journal of Control, Automation and Systems, vol. 7, no. 2, pp. 273-280, 2009.

[10] M. Zamani, M. Karimi-Ghartemani, N. Sadati, and M. Parniani, "Design of a fractional order PID controller for an AVR using particle swarm optimization," Control Engineering Practice, vol. 17, no. 12, pp. 1380-1387, 2009.

[11] D. H. Kim and J. H. Cho, "A biologically inspired intelligent PID controller tuning for AVR systems," International Journal of Control, Automation and Systems, vol. 4, no. 5, pp. 624-636, 2006.

[12] U. S. Banu and G. Uma, "Fuzzy gain scheduled continuous stirred tank reactor with particle swarm optimization based PID control minimizing integral square error," Instrumentation Science and Technology, vol. 36, no. 4, pp. 394-409, 2008.

[13] Y.-B. Wang, X. Peng, and B. Z. Wei, "A new particle swarm optimization based auto-tuning of PID controller," in Proceedings of the 7th International Conference on Machine Learning and Cybernetics, pp. 1818-1823, Kunming, China, July 2008.

[14] K. M. Passino, "Biomimicry of bacterial foraging for distributed optimization and control," IEEE Control Systems Magazine, vol. 22, no. 3, pp. 52-67, 2002.

[15] T. Jain and M. J. Nigam, "Optimization of PD-PI controller using swarm intelligence," International Journal of Computational Cognition, vol. 6, no. 4, pp. 55-59, 2008.
[16] A. Ali and S. Majhi, "Design of optimum PID controller by bacterial foraging strategy," in Proceedings of the IEEE International Conference on Industrial Technology (ICIT '06), pp. 601605, Mumbai, Indian, December 2006.

[17] D. H. Kim, "Hybrid GA-BF based intelligent PID controller tuning for AVR system," Applied Soft Computing Journal, vol. 11 , no. 1, pp. 11-22, 2011.

[18] D. H. Kim, A. Abraham, and J. H. Cho, "A hybrid genetic algorithm and bacterial foraging approach for global optimization," Information Sciences, vol. 177, no. 18, pp. 3918-3937, 2007.

[19] A. Biswas, S. Dasgupta, S. Das, and A. Abraham, "Synergy of PSO and bacterial foraging optimization-a comparative study on numerical benchmarks," in Advances in Soft Computing, vol. 44 of Innovations in Hybrid Intelligent Systems, pp. 255-263, 2007.

[20] S. V. R. S. Gollapudi, S. S. Pattnaik, O. P. Bajpai, S. Devi, and K. M. Bakwad, "Velocity modulated bacterial foraging optimization technique (VMBFO)," Applied Soft Computing Journal, vol. 11, no. 1, pp. 154-165, 2011.

[21] W. M. Korani, H. T. Dorrah, and H. M. Emara, "Bacterial foraging oriented by particle swarm optimization strategy for PID tuning," in Proceedings of the 8th IEEE International Conference on Computational Intelligence in Robotics and Automation (CIRA '09), pp. 445-450, Daejeon, Korea, December 2009.

[22] V. Rajinikanth and K. Latha, "Bacterial foraging optimization algorithm based pid controller tuning for time delayedunstable systems," The Mediterranean Journal of Measurement and Control, vol. 7, no. 1, pp. 197-203, 2011.

[23] C. T. Liou and C. Yu-Shu, "The effect of nonideal mixing on input multiplicity in a CSTR," Chemical Engineering Science, vol. 46, no. 8, pp. 2113-2116, 1991. 

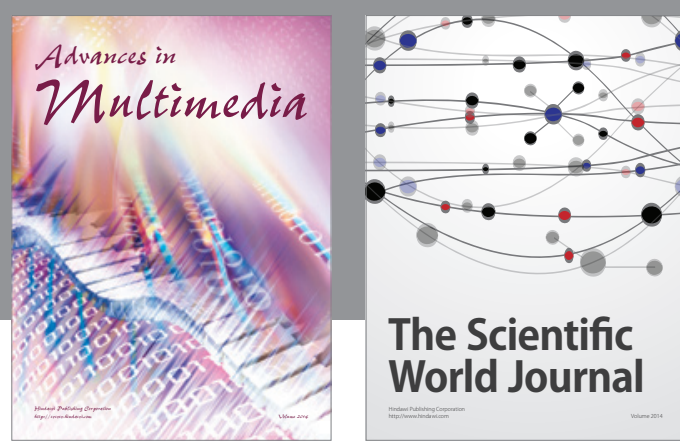

The Scientific World Journal
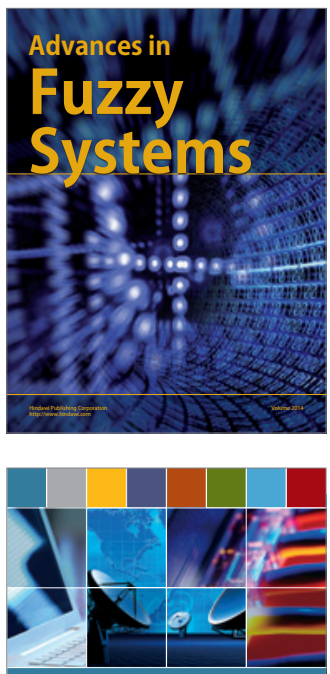

Computer Networks and Communications
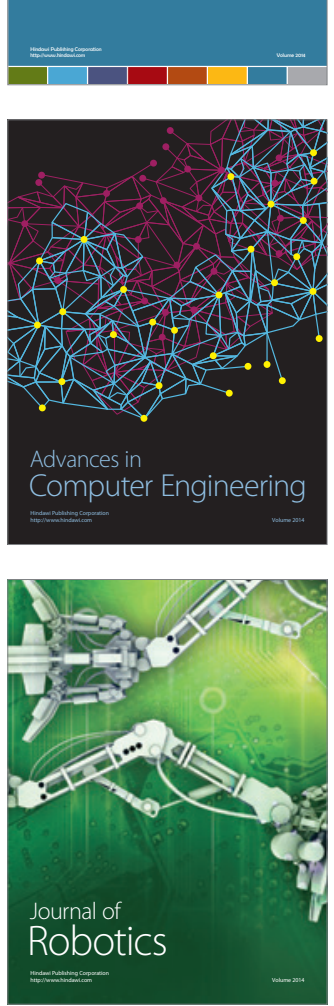
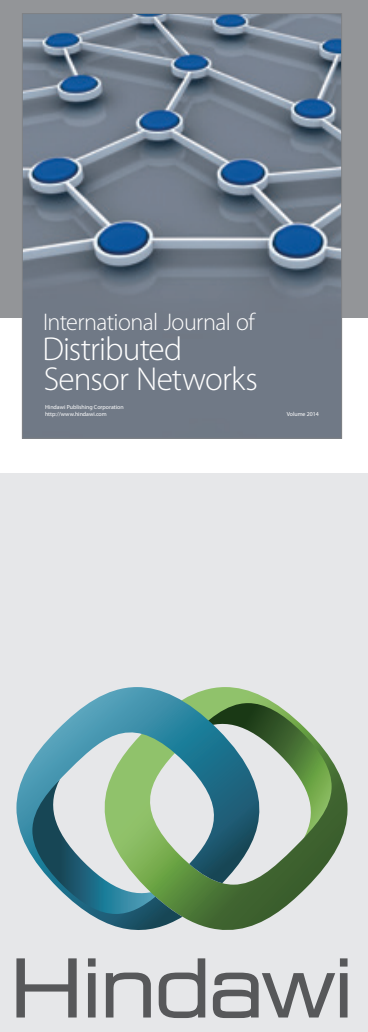

Submit your manuscripts at

http://www.hindawi.com
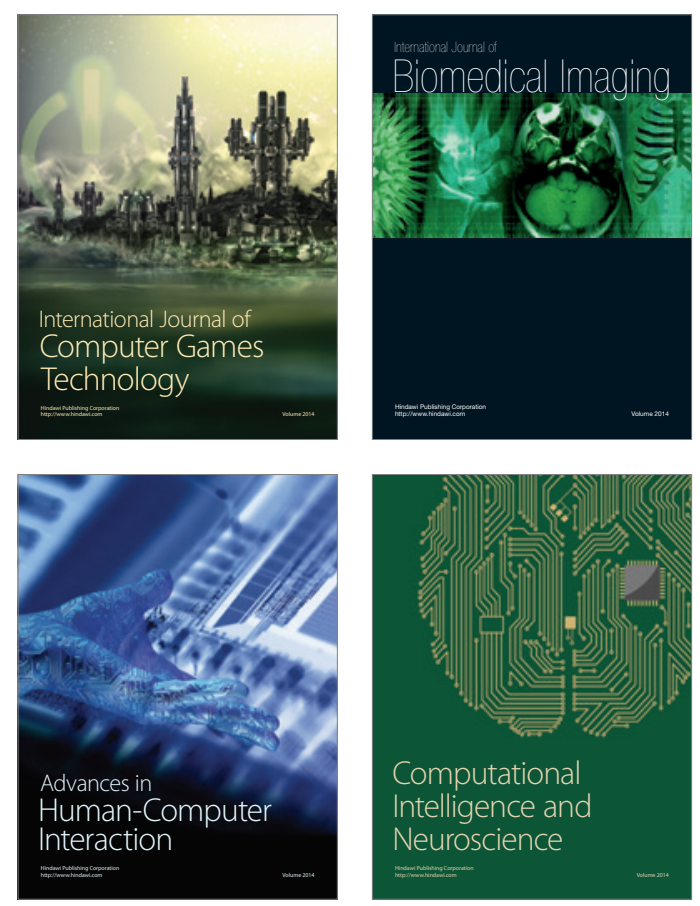
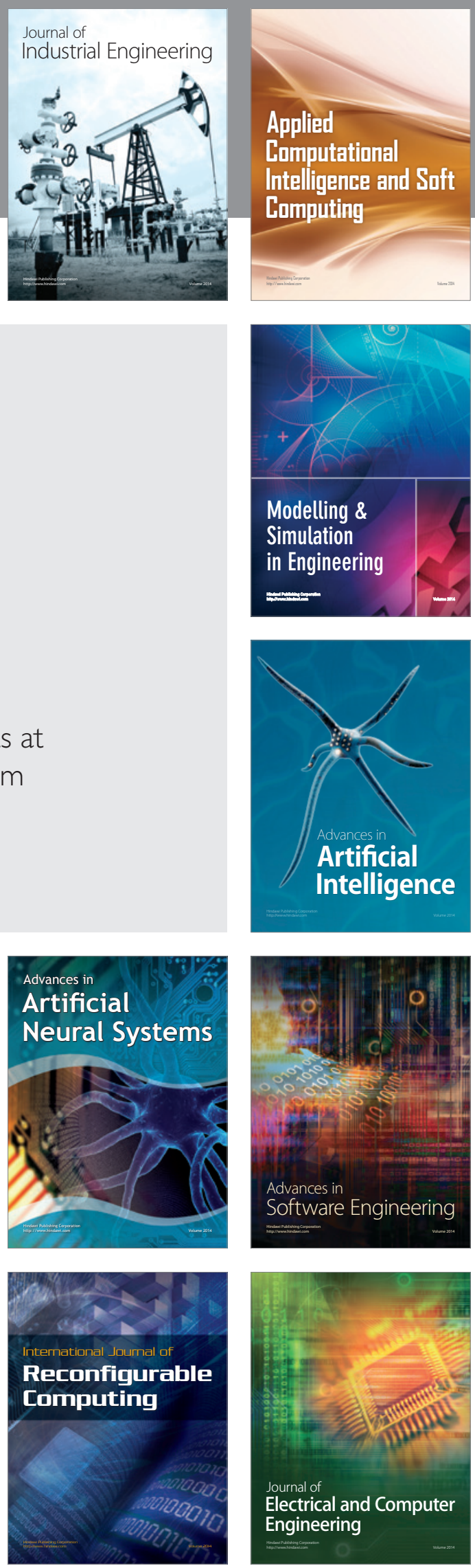